\title{
Original
}

\section{A New Experimental Mouse Model of Periodontitis Using an Orthodontic Ligature Wire}

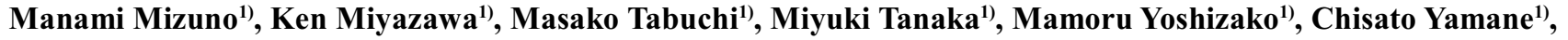 \\ Yasuyoshi Toriii ${ }^{1)}$, Hatsuhiko Maeda ${ }^{2)}$ and Shigemi Goto ${ }^{1)}$ \\ 1) Departments of Orthodontics, School of Dentistry, Aichi Gakuin University, Japan \\ 2) Departments of Oral Pathology, School of Dentistry, Aichi Gakuin University, Japan \\ (Accepted for publication, February 22, 2014)
}

\begin{abstract}
Periodontitis is characterized by alveolar bone resorption and loss of attachment. Recently, many induced periodontitis animal models have been developed to mimic alveolar bone resorption, but each model has a number of disadvantages. Periodontitis-inducing procedures should be simple and reproducible. Therefore, with the aim of establishing an experimental mouse model of periodontitis, we induced subgingival plaque accumulation in eight-week-old male C57BL/6 wild-type mice by placing an orthodontic ligature wire around the contact point between the left maxillary first and second molars. Maxillae were collected at four and eight weeks after ligature placement, and alveolar bone between the left molars (test maxillae) was compared with that between the right molars (control maxillae). Micro-computed tomography and pathological analysis were then performed to examine the degree of alveolar bone resorption. Alveolar bone resorption was significant in the test maxillae compared with the controls. In addition, the ligature wire was not found to affect the periodontal tissue. The induction of alveolar bone resorption was likely due to the direct mechanical impact (mechanical irritation of gingiva) of the wire ligature. These findings suggest that placement of the ligature wire around the contact point between teeth successfully induced alveolar bone resorption. Furthermore, use of the ligature wire removed the need to induce alveolar bone resorption by using an impacted wire ligature to create mechanical stress. Therefore, the present report provides a new animal model of periodontitis.
\end{abstract}

Key words: Periodontitis, Mouse model, Micro-CT, Ligature wire

\section{Introduction}

In recent years, a growing number of middle-aged and advanced-age adults with difficulty in brush like crowding case have opted for orthodontic treatment as a preventive therapy for periodontitis resulting from the accumulation of food residues. However, in many of these patients, the periodontium has already deteriorated due to periodontal disease. Despite the necessity of preventing alveolar bone resorption in such patients, no practical means of inhibition is available, so experimental models of periodontitis play a pivotal role in the development of inhibitors of alveolar bone resorption in periodontitis. Several studies have reported periodontitis in experimental animals and have focused on induction of periodontitis by persistent bacterial challenge via repeated administration of bacteria, bacterial components, or bacterial products (oral gavage model) ${ }^{1-6)}$ or creation of a

Correspondence to: Dr. Manami Mizuno, Departments of Orthodontics, School of Dentistry, Aichi Gakuin University, 2-11 Suemoridoori Chikusa-ku Nagoyashi, Aichi, 464-8651 Japan; Tel: +81-52-7592111(5378); Fax: +81-52-751-8900; E-mail: manamin@dpc.agu.ac.jp subgingival environment prone to food impaction by using modifying devices, such as cotton ligatures ${ }^{7-12}$. However, many of these experimental models have disadvantages when establishing induced periodontitis. The oral gavage model changes oral flora. The alteration of the endogenous flora may contribute to the disease process rather than the direct action of the bacteria. And the conventional ligature placing method, wherein ligatures are placed around a tooth crown, is associated with direct mechanical irritation on the gingiva. To address this disadvantage, we tested ligature placement at the contact point between teeth. We hypothesize that this experimental model could induced periodontitis caused by food impaction and avoid direct mechanical irritation of gingiva.

\section{Materials and Methods}

\section{Experimental animals and reagents}

C57BL/6 wild-type mice were purchased from CLEA Japan (Tokyo, Japan) and maintained in the animal facility of the School of Dentistry of Aichi Gakuen University under the following 

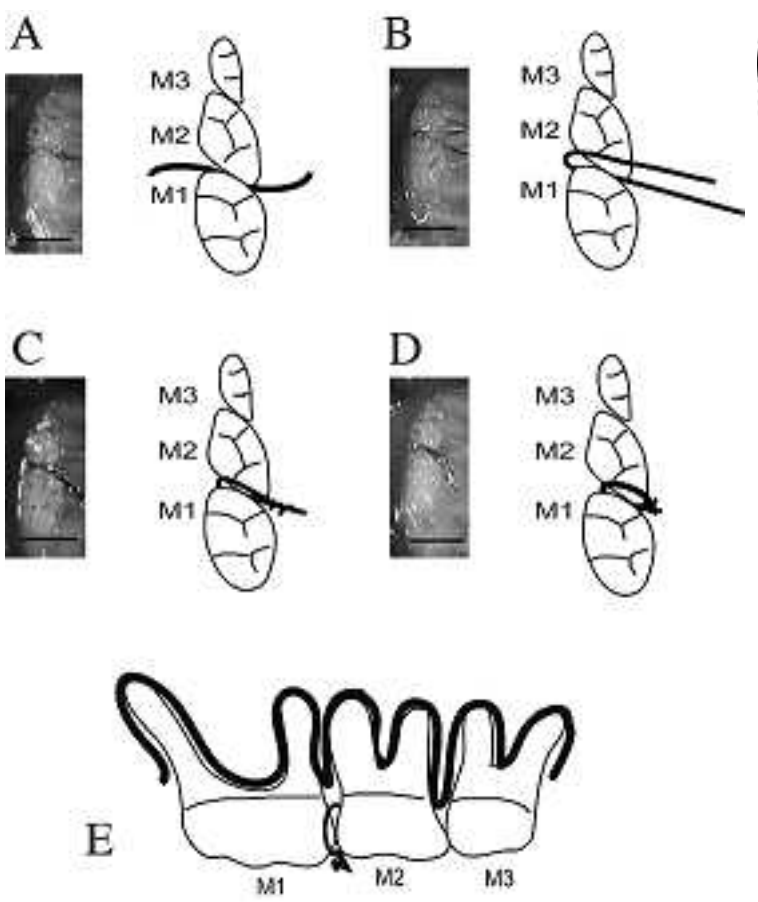

Figure 1. Technical procedures of ligation.M1; firstmolar, M2; second Molar, M3; third molar (Scale bar: $1500 \mu \mathrm{m}$ )

A: A 0.1-mm-diameter stainless steel ligature wire was passed through interdentium between first molar and second molar. B: Ligature was turned down to lingual side.

C: Ligature was looped around contact point.

D: Ligature was tied and excess ligature was cut.

E: Position of ligature wire placement (Buccal view)

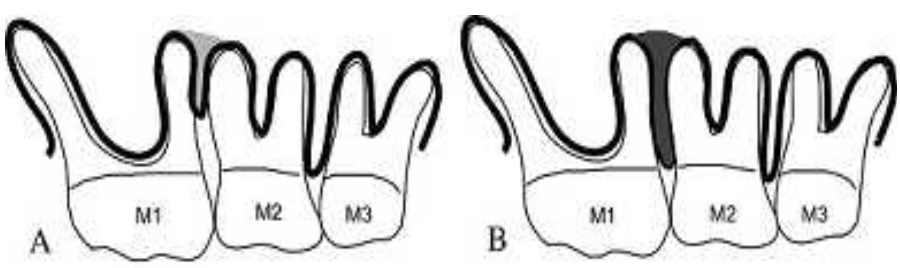

C Formular for calculating Space between the

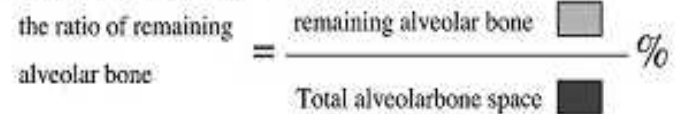

Figure 2.

A: Shematic diagram of the space between the remaining alveolar bone B: Shematic diagram of the total alveolar bone space

$\mathrm{C}$ : Formula for calculating the ratio of remaining alveolar bone

at $90 \mathrm{kv}$ and $88 \mu \mathrm{A}$ for $2 \mathrm{~min}$. The degree of alveolar bone resorption was analyzed according to the method reported by Park et al. ${ }^{13)}$, with modification. Briefly, alveolar bone volume between M1 and M2 was measured using TRI/3D-BON software (Ratoc System Engineering Co., Ltd, Tokyo, Japan). The total alveolar bone space was defined as the space with a height from the cementoenamel junction line to the root apex line. The ratio of the remaining alveolar bone was calculated by dividing its volume by the total alveolar bone volume (Fig. 2), and the ratio of the remaining alveolar bone between the left M1 and M2 (test maxillae) was compared with that in the right maxilla (control maxillae).

\section{Pathological analysis}

Collected maxillae were fixed in $10 \%$ neutral buffered formalin and decalcified in $10 \%$ EDTA ( $\mathrm{pH} 7.2)$ at $4{ }^{\circ} \mathrm{C}$ for approximately four weeks. Paraffin blocks were prepared according to the standard method and cut into 5- $\mu$ m-thick mesiodistal serial sections. After that, there were stained with hematoxylin-eosin (HE).

\section{Statistical analysis}

Data are expressed as mean \pm standard deviation values. Statistical significance was assessed using Mann Whitney test.

\section{Results}

Micro CT findings and changes in the ratio of remaining

\section{alveolar bone}

Micro CT showed gradual alveolar bone resorption during the test period in the test maxillae, but no marked resorption was observed in the controls (Fig. 3 a-f), and more, the ligatures were placed contactpoints and their didn't touch gingiva. The ratio of remaining alveolar bone was significantly lower at four weeks ( $p$ $<0.01)$ and eight weeks $(p<0.01)$ after ligature placement (Fig. $4)$, suggesting significant alveolar bone resorption in response to 
Manami Mizuno et al.: Ligature Wire Periodontitis Mouse Model
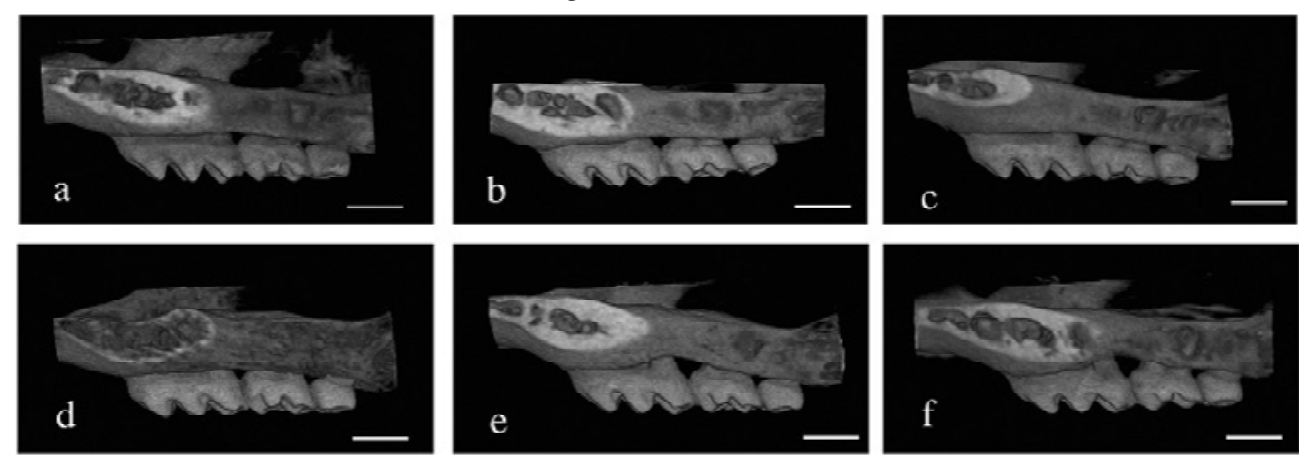

Figure 3. Micro-computed tomography images of the alveolar bone of test and control maxillae (buccal view) (Scale bar: $1000 \mu \mathrm{m}$ ) a: The no-ligature sides at 0 week b: The no-ligature sides at 4 weeks $\quad$ c: The no-ligature sides at 8 weeks d: The ligature sides at 0 week $\quad$ e: The ligature sides at 4 weeks $\quad f$,:The ligature sides at 8 weeks

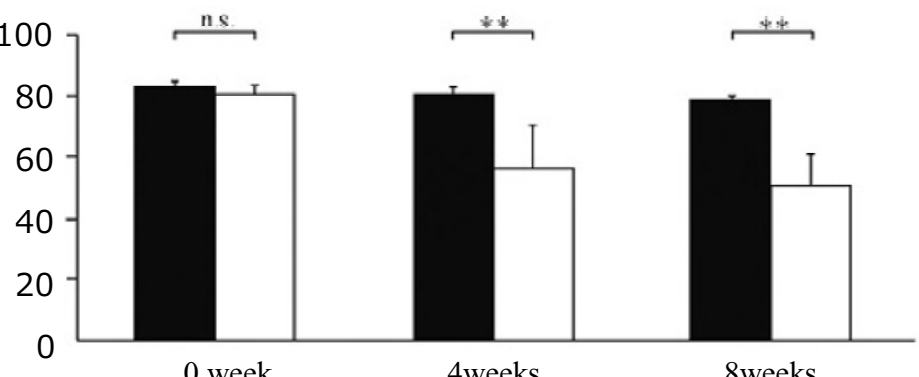

— ligature sides

$\square$ controls
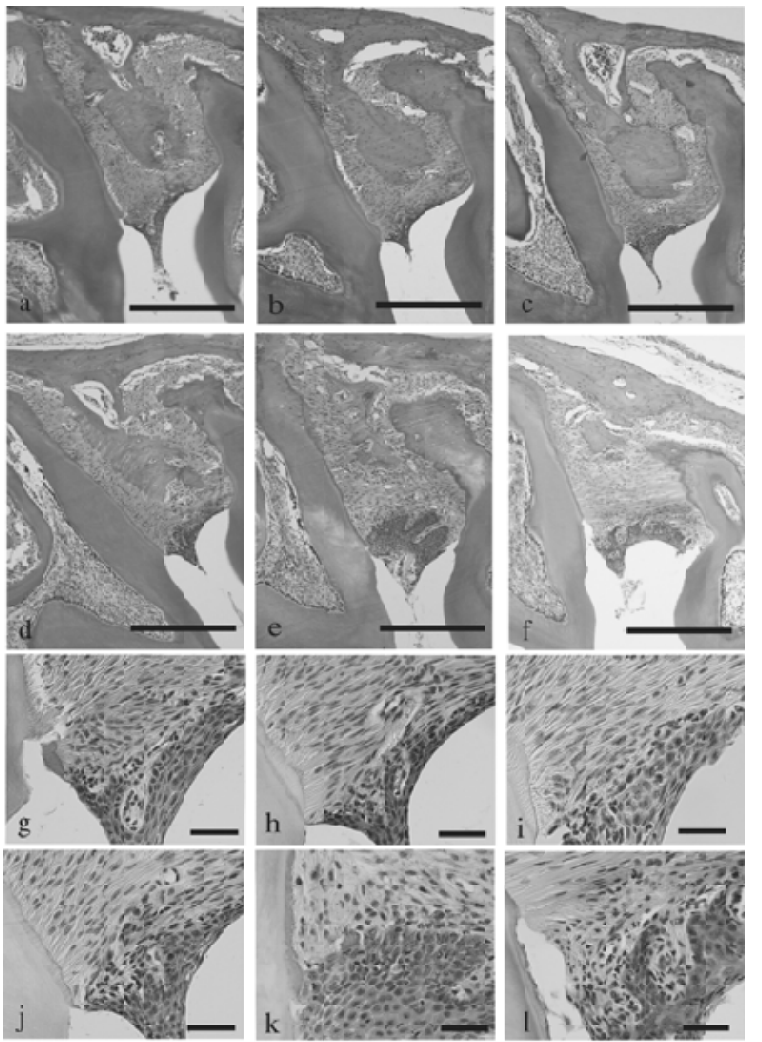

ligature placement.

\section{Pathohistological findings}

In the control maxillae, alveolar bone height was preserved,
Figure 5 .

Hematoxylin-eosin stained periodontium between the first and second molars. (Scale bar: $500 \mu \mathrm{m}$ )

a: The no-ligature sides at 0 week

b: The no-ligature sides at 4 weeks

c: The no-ligature sides at 8 weeks

$\mathrm{d}$ : The ligature sides at 0 week

e: The ligature sides at 4 weeks

f: The ligature sides at 8 weeks

Hematoxylin-eosin stained periodontium around the alveolar crestal region between the first and second molars. (under the gingival epithelium) (Scale bar: $50 \mu \mathrm{m}$ )

$\mathrm{g}$ : The no-ligature sides at 0 week

$\mathrm{h}$ : The no-ligature sides at 4 weeks

i: The no-ligature sides at 8 weeks

$\mathrm{j}$ : The ligature sides at 0 week

$\mathrm{k}$ : The ligature sides at 4 weeks

1: The ligature sides at 8 weeks

thickening of the cornified layer of the gingiva was absent, and no abnormalities were found in the periodontium at either time point (Fig. 5 a-c). On the other hand, in the test maxillae, signs of gingivitis, such as infiltration of inflammatory cells and thickening 
of the cornified layer, were observed with attachment loss and bone resorption at both time points, but these signs were particularly prominent at eight weeks after ligature placement (Fig. 5-f). High-magnification images showed no signs of inflammation in the control maxillae. More precisely, the alveolar bone was surrounded by fibroblasts, and the periodontal ligament exhibited regular fiber orientation (Fig. 5 g-i). On the other hand, infiltration of inflammatory cells (mainly neutrophils), apical elongation of junctional eptheliums and dilated capillaries were observed in the test maxillae at both four weeks and eight weeks after ligature placement. Furthermore, destruction of gingival fibers were observed in it at eight weeks after the experiment (Fig. 5 j-1).

\section{Discussion}

At present, experimental periodontitis is often induced by simulating plaque retentive conditions, for example, by placing plaque retentive cotton ligatures around the tooth crown ${ }^{14)}$. We previously attempted placement of an orthodontic ligature wire around the crown of a left maxillary first molar to induce periodontitis. However, the wire slid and encroached on the gingiva, probably because the crown of the mouse molar is funnelshaped, suggesting that direct stimulation by the wire contributed to the induction of bone resorption. Similar mechanical stimulation might have played a role in the study reported by Jin et al. who induced periodontitis by placing cotton ligatures in the gingival sulcus ${ }^{14)}$. Yoshinari et al. attempted placement of a loose cotton ligature between the right maxillary first and second molars for nine weeks ${ }^{15}$. However, in their model, the loose ligature might have pushed the gingiva while the ligature was settling during the experimental period, which, in addition to food impaction, might have caused periodontitis directly ${ }^{15}$. Thus, in the present study, we attempted a new means of ligature placement that comprised tying the ligature around the contact point between the first and second molars. This method successfully retained the ligature in place, thereby preventing direct ligature-gingiva contact and resulting mechanical stimulation. Furthermore, we believe that this method impairs the self-clearing function and that captured food residues caused inflammation and induced experimental periodontitis. Moreover, because of the small diameter and good fit of the wire with the contact point, it is unlikely that the wire added to the orthodontic force. But our study is noteworthy as $80 \%$ of the placed ligature wires remained at their original contact point, implying high potential for reproducibility.

Xie et al. ${ }^{16)}$ and Jin et al. ${ }^{14)}$ showed ligature model, but our model different from their models. Jin et al. ${ }^{14)}$ described that ligatures displaced apically into the gingival sulci to ensure a subgingival position. But our experimental period the ligatures were placed contactpoints and their didn't touch gingiva.

Many factors, including administration of agents, will be assessed in further research on periodontitis treatment.
Experimental periodontitis should be induced in a simple and reproducible manner, otherwise the experimental model itself will become unreliable, threatening the credibility of study findings. The present method involves a single step tying a ligature wire around the contact point between the first and second molars which can avoid direct injury and is thus an effective model for studying the pathology of periodontitis induced by food impaction.

\section{Acknowledgements}

This study was supported by a Grand-in-Aid for Scientific Research from the Ministry of Education, Science and Culture of Japan (24593115).

\section{References}

1. Okada Y, Hamada N, Kim Y, Takahashi Y, Sasaguri K, Ozono $\mathrm{S}$ and Sato S. Blockade of sympathetic â-receptors inhibits Porphyromonas gingivalis-induced alveolar bone loss in an experimental rat periodontitis model. Arch Oral Biol 55: 502508,2010

2. Polak D, Wilensky A, Shapira L, Halabi A, Goldstein D, Weiss EI and Houri-Haddad Y. Mouse model of experimental periodontitis induced by Porphyromonas gingivalis/ Fusobacterium nucleatuminfection: bone loss and host response. J Clin Periodontol 36: 406-410, 2009

3. Vardar S, Buduneli E, Baylas H, Berdeli AH, Buduneli N and Atilla G. Individual and combined effects of selective cyclooxygenase- 2 inhibitor and omega- 3 fatty acid on endotoxin-induced periodontitis in rats. J Periodontol 76: 99-106, 2005

4. Hamada N, Watanabe K, Tahara T, Nakazawa K, Ishida I, Shibata Y, Kobayashi T, Yoshie H, Abiko Y and Umemoto $\mathrm{T}$. The $\mathrm{r} 40-\mathrm{kDa}$ outer membrane protein human monoclonal antibody protects against Porphylomonas gingivalis-induced bone loss in rats. J Periodontal 78: 933-939, 2007

5. Sugiyama S, Takahashi S, Tokutomi F, Yoshida A, Kobayashi K, Yoshino F, Wada-Takahashi S, Toyama T, Watanabe K, Hamada N, Todoki K and Lee MC. Gingival vascular functions are altered in type 2 diabetes mellitus model. J Clin Biochem Nutr 51: 108-113, 2012

6. Yoshino F, Yoshida A, Wada-Takahashi S, Sugiyama S, Tokutomi F, Maehata Y, Miyamoto C, Komatsu T, Takahashi S, Kobayashi K and Lee MC. Assessments of salivary antioxidant activity using electron spin resonance spectroscopy. Arch Oral Biol 57: 654-662, 2012

7. Luan Q, Desta T, Chehab L, Sanders VJ, Plattner J and Graves DT. Inhibition of experimental periodontitis by a topical boron-based antimicrobial. J Dent Res 87: 148-152, 2008

8. Goya JA, Paez HA and Mandalunis PM. Effect of topical administration of monosodium olpadronate on experimental 
Manami Mizuno et al.: Ligature Wire Periodontitis Mouse Model

periodontitis in rats. J Periodontol 77: 1-6, 2006

9. Caton JG and Zander HA. Primate model for testing periodontal treatment procedures: I. Histologic investigation of localized periodontal pockets produced by orthodontic elastics. J Periodontol 46: 71-77, 1975

10. Nishikawa T, Naruse K, Kobayashi Y, Miyajima S, Mizutani M, Kikuchi T, Soboku K, Nakamura N, Sokabe A, Tosaki T, Hata M, Ohno N, Noguchi T and Matsubara T. Involvement of nitrosative stress in experimental periodontitis in diabetic rats. J Clin Periodontol 39: 342-349, 2012

11. Branco-de-Almeida LS, Franco GCN, Castro ML, Santos JG, Anbinder AL, Cortelli SC, Kajiya M, Kawai M and Rosalen PL. Fluoxetine inhibits inflammatory response and bone loss in a rat model of ligature-induced periodontitis. J Periodontol 83: 664-671, 2012

12. de Menezes AMA, de Souza GFP, Gomes AS, de Carvalho Leitao RF, de Albuquerque Ribeiro R, de Oliveria MG and de Castro Brito A. S-nitrosoglutathione decreases inflammation and bone resorption in experimental periodontitis in rats. J Periodontol 83: 514-521, 2012

13. Park CH, Abramson ZR, Taba MJ, Jin JQ, Chang J, Kreider JM, Goldstein SA and Giannobile WV. Three-dimensional micro-computed tomographic imaging of alveolar bone in experimental bone loss or repair. J Periodontol 78: 273-281, 2007

14. Jin Q, Cirelli JA, Park CH, Sugai JV, Taba MJ, Kostenuik PJ, and Giannobile WV. RANKL Inhibition through osteoprotegerin blocks bone loss in experimental periodontitis. J Periodontol 78: 1300-1308, 2007

15. Yoshinari N, Kameyama Y, Aoyama Y, Nishiyama H and Noguchi T. Effect of long-term methotrexate induced neutropenia on experimental periodontal lesion in rats. J Periodontal Res 29: 393-400, 1994

16. Xie R, Kuijpers-jagtman AM and Maltha JC. Inflammatory responses in two commonly used rat models for experimental tooth movement: Comparison with ligature-induced periodontitis. Arch Oral Biol 56: 159-167, 2011 
J.Hard Tissue Biology Vol. 23(2):255-260, 2014 